Pacific Journal of Mathematics

MULTIPLIERS ON A BANACH ALGEBRA WITH A BOUNDED 


\title{
MULTIPLIERS ON A BANACH ALGEBRA WITH A BOUNDED APPROXIMATE IDENTITY
}

\author{
JoHN W. DAVENPORT
}

\begin{abstract}
Let $A$ be a Banach algebra with a bounded approximate identity $\left\{e_{\alpha} \mid \alpha \in \Lambda\right\}$, and $M(A)$ the multiplier algebra on $A$. In this paper, we obtain a representation for $M(A)$ such that each multiplier operator appears as a multiplicative operator. The proof makes use of the weak-* compactness of the net $\left\{T e_{\alpha} \mid \alpha \in \Lambda\right\}$ and the algebraic properties of a multiplier.
\end{abstract}

1. Introduction. In 1951, J.G. Wendel showed that the left centralizers on $L_{1}(G), G$ a locally compact group, was equivalent to $C_{0}(G)^{*}$, the space of regular Borel measures on $G$. Thus, if $T$ is a centralizer and $x$ is any element in $L_{1}(G)$ then $T x=\xi * x$ for some Borel measure $\xi$. It is also well known that if $A$ is a Banach algebra with an identity element then any multiplier on $A$ is determined by its action on the identity element. In this paper, we show that if $A$ is a Banach algebra with a bounded approximate identity then there exist a continuous isomorphism of $A$ such that each multiplier defined on $A$ is given by point-wise multiplication. In the case that the approximate identity is uniformly bounded by one, the representation is norm preserving. Thus we obtain an isometric isomorphism for all multipliers on $L_{1}(G)$ and for all multipliers on any $B^{*}$-algebra such that the action of a multiplier is given by point-wise multiplication by a fixed element in $A$.

\section{The representation space for $M(A)$.}

Definition 2.1. Let $A$ be a Banach algebra and $T$ a mapping from $A$ into $A$. The map $T$ is a multiplier provided

$$
x(T y)=(T x) y \quad(x, y \in A) .
$$

Every multiplier turns out to be a continuous function and the set of all multipliers on $A$ under pointwise operations is a commutative subalgebra of $B(A)$, the set of all bounded linear operators on $A([5])$.

Notation 2.2. In this paper, a Banach algebra with a bounded approximate identity will be denoted by $A$ and the multiplier algebra on $A$ will be denoted by $M(A)$. For any Banach algebra $X$, we denote the weak-* convergence of a net in $X^{*}$, the dual space of $X$, indexed by $\alpha \in \Lambda$, by " $\lim _{\alpha}^{w k-*}(\cdot)$ ". Unless otherwise stated, we denote the bound on the approximate identity by $M$. 
Definition 2.3. Let $X$ be a Banach algebra. The algebra $X$ is said to have a bounded approximate identity provided there exists a net $\left\{e_{\alpha} \mid \alpha \in \Lambda\right\}$ in $X$ and a $M>0$ such that

2.3.1 $\left\|e_{\alpha}\right\|<M$

2.3.2 $\lim _{\alpha} e_{\alpha} x=\lim _{\alpha} x e_{\alpha}=x$ $(x \in X)$.

DEFINITION 2.4. Let $\left\{e_{\alpha} \mid \alpha \in \Lambda\right\}$ denote the approximate identity on $A$, and $B_{*}=\left\{f \in A^{*} \mid f \cdot e_{\alpha} \rightarrow f\right\}$ where $f \cdot a(x)=f(a x)$ for each a, $x \in A$ and $f \in A^{*}$. The set $B_{*}$ is a closed subspace of $A^{*}$ and $B_{*}=$ $\left\{f \cdot a \mid f \in A^{*}, a \in A\right\}$ ([3]). By defining
2.4.1 $[G, f]=G(f \cdot a)$
$\left(a \in A, f \in B_{*}, G \in B_{*}^{*}\right)$
2.4.2 $F \cdot G(f)=F[G, f]$
$\left(f \in B_{*}, F, G \in B_{*}^{*}\right)$,

the dual space, $B_{*}^{*}$, becomes a Banach algebra. This follows since the above definitions are the restrictions to $B_{*}$ of the Arens product on $A^{* *}$ which makes $A^{* *}$ into a Banach algebra such that if $\pi$ is the canonical embeding of $A$ into $A^{* *}$ then $\pi$ is an isometric isomorphism ([5]).

LEMMA 2.5. There exists a norm reducing isomorphism of $A$ into $B_{*}^{*}$.

Proof. We define $\tau: A \rightarrow B_{*}^{*}$ by $\tau a(f)=f(a)=\left.\pi \alpha\right|_{B^{*}}$.

Clearly $\tau$ is linear and since $B_{*}=\left\{f \cdot a \mid f \in A^{*}, a \in A\right\}$, it follows that $\tau$ is one-to-one. From $|\tau a(f)|=|f(a)|<\|f\| \cdot\|a\|$, we see that $\|\tau a\|<\|a\|$, for all $a \in A$.

Lemma 2.6. Let $\left\{F_{\alpha} \mid a \in \Lambda\right\}$ be a net in $B_{*}^{*} ; a \in A$; and $F, G \in B_{*}^{*}$, then the following properties are satisfied:

2.6.1 if $\lim _{\alpha}^{w k-*} F_{\alpha}=F$ then $\lim _{\alpha}^{w k-*} F_{\alpha} \cdot G=F \cdot G$

2.6.2 if $\lim _{\alpha}^{w k-*} F_{\alpha}=F$ then $\lim _{\alpha}^{w k-*} \tau a \cdot F_{\alpha}=\tau a \cdot F$

2.6.3 if $F \cdot \tau a=0$ for all $a \in A$ or $\tau a \cdot F=0$ for all $a \in A$ then $F=0$.

Proof. These properties follow from a straightforward application of the definitions of the operations involved.

Lemma 2.7. The Banach algebra $B_{*}^{*}$ has an identity element which we denote by $J$.

Proof. From $\left\|\tau e_{\alpha}\right\|<\left\|e_{\alpha}\right\|<M$, it follows that the net $\left\{\tau e_{\alpha}\right\}$ has a weak-* convergent subnet. Let $J=\lim _{\alpha}^{w k-t} \tau e_{\alpha}$. Since

$$
[J, f](x)=J(f \cdot x)=\lim _{a} \tau e_{a}(f \cdot x)=\lim _{a} f\left(x e_{\alpha}\right)=f(x),
$$


for all $x \in A$, we have that $[J, f]=f$ for all $f \in B_{*}$. Thus $F \cdot J=F$, for all $F \in B_{*}^{*}$. Since $\tau a \cdot F$ is weak-* continuous in $F$, it also follows that $J \cdot F(f)=\lim _{\alpha} \tau e_{\alpha} \cdot F(f)=\lim _{\alpha} F\left(f \cdot e_{\alpha}\right)=F(f)$ for all $f \in B_{*}$ and $F \in B_{*}^{*}$. Thus $J \cdot F=F$ for all $F \in B_{*}^{*}$.

THEOREM 2.8. Let $A$ be a Banach algebra with a bounded approximate identity $\left\{e_{\alpha} \mid \alpha \in \Lambda\right\}$. Then there exists a map $\mu$ from $M(A)$ into $B_{*}^{*}$ such that $\mu$ is a continuous, algebraic isomorphism of $M(A)$ into $B_{*}^{*}$. Furthermore

$$
\tau(T a)=(\mu T) \cdot \tau \alpha=\tau \alpha \cdot(\mu T) \quad(\alpha \in A, T \in M(A)) .
$$

Proof. Let $T \in M(A)$. Since $\left\|T e_{\alpha}\right\|<\|T\| \cdot M$, the net $\left\{\tau\left(T e_{\alpha}\right) \mid \alpha \in \Lambda\right\}$ has a weak-* convergent subnet in $B_{*}^{*}$. If $\left\{\tau\left(T e_{\beta}\right) \mid \beta \in \Gamma\right\}$ converges to $G$ and $\left\{\tau\left(T e_{\alpha}\right) \mid \alpha \in \Lambda\right\}$ converges to $F$, each in the weak-* topology; then, for each $f \in B_{*}$, we have that

$$
\begin{aligned}
F(f) & =\lim _{\alpha} \tau\left(T e_{\alpha}\right)(f)=\lim _{\alpha} \tau\left(T e_{\alpha}\right) \cdot J(f) \\
& =\lim _{\alpha} \lim _{\beta} \tau T e_{\alpha} \cdot \tau e_{\beta}(f)=\lim _{\alpha} \lim _{\beta}\left(\tau T e_{\alpha}\left(e_{\beta}\right)\right)(f) \\
& =\lim _{\alpha} \lim _{\beta} \tau e_{\alpha} \cdot \tau T e_{\beta}(f)=\lim _{\alpha} \tau e_{\alpha} \cdot G(f)=G(f) .
\end{aligned}
$$

Now we define the mapping $\mu$ from $M(A)$ to $B_{*}^{*}$ by

$$
\mu(T)=F=\lim _{\alpha}^{w k-*} \tau\left(T e_{\alpha}\right) \quad(T \in M(A)) .
$$

The previous remarks show that $\mu$ is well defined. We first observe that if $F=\mu(T)$, then

$$
\tau a \cdot F(f)=\lim _{\alpha} \tau a \cdot \tau T e_{\alpha}(f)=\lim _{\alpha} \tau T a \cdot \tau e_{\alpha}(f)=\tau(T a)(f) .
$$

Thus

2.8.1. $\tau a \cdot \mu(T)=\tau(T a)$

$(a \in A, T \in M(A))$.

By Lemma 2.7, the identity element of $B_{*}^{*}$ is the weak-* limit of a subnet of $\left\{\tau e_{\alpha} \mid \alpha \in \Lambda\right\}$. Let $\left\{\tau e_{\beta}\right\}$ denote this subnet. Hence we have

$$
\begin{aligned}
\mu(T) \cdot \tau \alpha(f) & =\lim _{\beta} \tau e_{\beta} \cdot \mu(T) \cdot \tau \alpha(f)=\lim _{\beta} \tau e_{\beta} \cdot \mu(T) \cdot \tau \alpha(f) \\
& =\lim _{\beta} \tau e_{\beta} \cdot \tau T a(f)=\tau T a(f) .
\end{aligned}
$$

Therefore,

2.8.2. $\mu T \cdot \tau a=\tau T a$ $(a \in A, T \in M(A))$.

Let $x, y \in A$ and $T \in M(A)$. Then 


$$
\begin{aligned}
\tau x \cdot \mu(T S) \cdot \tau y & =\tau(T S x) y=\tau S x \cdot \tau T y=\mu S \cdot \tau x \cdot \mu T \cdot \tau y \\
& =\tau x \cdot \mu S \cdot \mu T \cdot \tau y
\end{aligned}
$$

and thus by Lemma 2.6, it follows that $\mu(T S)=\mu(S) \cdot \mu(T)$. But C. N. Kellogg [4] proved that $M(A)$ is a closed commutative subalgebra of $B(A)$, the set of all bounded linear operators on $A$. Thus $\mu(T S)=\mu(S T)=\mu(T) \cdot \mu(S)$ and therefore $\mu$ is homomorphic.

If $\mu(T)=\mu(S)$ for some $T, S \in M(A)$ where $\mu(T)=\lim _{\alpha}^{w k-*} \tau T e_{\alpha}$ and $\mu(S)=\lim _{\beta}^{w k-*} \tau S e_{\beta}$ then for each $f \in B_{*}$, and $a \in A$, we have

$$
\begin{aligned}
\tau(T a)(f) & =\lim _{\alpha} \tau(T a) \cdot \tau e_{\alpha}(f)=\lim _{\alpha} \tau \alpha \cdot \tau T e_{\alpha}(f) \\
& =\tau \alpha \cdot \mu(T)(f)=\tau a \cdot \mu(S)(f)=\tau \alpha \cdot \lim _{\beta} \tau\left(S e_{\beta}\right)(f) \\
& =\lim _{\beta} \tau \alpha \cdot \tau\left(S e_{\beta}\right)(f)=\lim _{\beta} \tau(S \alpha) \cdot e_{\beta}(f)=\tau(S \alpha)(f) .
\end{aligned}
$$

Since $\tau$ is one-to-one, it follows that $T a=S a$ for each $a \in A$. Thus $\mu$ is one-to-one.

From $\mu(T)=\lim _{\alpha} \tau T e_{\alpha}$ and $\left\|\tau T e_{\alpha}\right\|<\left\|T e_{\alpha}\right\|<\|T\| \cdot\left\|e_{\alpha}\right\|<$ $\|T\| \cdot M$, it follows that $\mu$ is continuous.

CoROLlaRY 2.9. If $M=1$, then $M(A)$ is isometrically *-isomorphic to a subspace of $B_{*}^{*}$.

Proof. This follows from Theorem 2.8 and the fact that $\|\tau a\|=\|a\|$.

For $A=L_{1}(G), G$ a nondiscrete locally compact abelian group, the space $B_{*}$ is the space of uniformily continuous bounded functions on $G$ and $B_{*}^{*}$ is the space $M(G)$ of bounded measures of the maximal ideal space of $B_{*}$. If $G$ is compact then $M(A)=M(G)$. In the case that $A$ is a $B^{*}$-algebra, we have the following result.

COROLlaRY 2.10. If $A$ is a $B^{*}$-algebra then $M(A)$ is isometrically $*$-ismorphic to a subspace of $A^{* *}$. Furthermore, if $\mu(T)=F$ for $T \in M(A)$ and $F \in A^{* *}$, then

$$
\pi a \cdot F=F \cdot \pi a=\pi T a
$$

where the above operation is the Arens product on $A^{* *}$.

Proof. D. C. Taylor [7] has shown that $A^{*}=\left\{f \cdot a \mid f \in A^{*}, a \in A\right\}=$ $\left\{a \cdot f \mid f \in A^{*}, a \in A\right\}$. Thus $B_{*}=A^{*}$ and $B_{*}^{*}=A^{* *}$. In this case the product operation on $B_{*}^{*}=A^{* *}$ becomes the Arens product and the involution on $A^{* *}$ is given by $F^{*}(f)=\overline{F(\bar{f})}$ where $\bar{f}\left(x^{*}\right)$ [2]. Since a $B^{*}$-algebra possesses an approximate identity uniformly bounded by one, the result follows from Corollary 2.9. 
CoRollary 2.11. Let $A$ be a $B^{*}$-algebra. Then $F \in A^{* *}$ belongs to $\mu(M(A))$ if and only if the operator $F$ commutes with $\pi A$ and $F \cdot \pi a$ is continuous in the weak-* topology on $A^{*}$ for each $a \in A$.

The author wishes to thank Professor C. N. Kellogg for his encouragement and guidance.

\section{REFERENCES}

1. R. Arens, Operations induced in function classes, Monat. fur Math., 55 (1951), $1-19$.

2. F. F. Bonsall, and J. Duncan, Numerical Ranges of Operators on Normed Spaces and of Elements of Normed Algebras, London Math. Soc. Lecture Notes, Series II, London, 1971.

3. P. Curtis and Figa-Talamanca, Factorization theorems for Banach algebras in Function Algebras, edited by F. T. Birtel, Scott, Foresman and Co., Chicago, Ill., 1966, 169-185.

4. C. N. Kellogg, Centralizers and $H^{*}$-algebras, Pacific J. Math., 17 (1966), 121-129.

5. R. Larson, An Introduction to the Theory of Multipliers, Springer, New York, 1971.

6. C. E. Rickart, General Theory of Banach Algebras, Van Nostrand, Princeton, 1960.

7. D. C. Taylor, The strict topology for double centralizer algebras, Trans. Amer. Soc., 150 (1970), 633-643.

8. J. G. Wendel, Left centralizers and isomorphisms of group algebras, Pacific J. Math., 2 (1952), 251-261.

Received March 18, 1975 and in revised form December 8, 1975.

Madison College, Harrisonburg, VA 



\section{PACIFIC JOURNAL OF MATHEMATICS}

EDITORS

RICHARD ARENS (Managing Editor)

University of California

Los Angeles, California 90024

R. A. BEAUMONT

University of Washington

Seattle, Washington 98105
J. DugundJI

Department of Mathematics

University of Southern California

Los Angeles, California 90007

D. Gilbarg and J. Milgram

Stanford University

Stanford, California 94305

\section{ASSOCIATE EDITORS}

E. F. BECKENBACH

B. H. NeumanN

F. WOLF

K. YosHIDA

\section{SUPPORTING INSTITUTIONS}

UNIVERSITY OF BRITISH COLUMBIA

UNIVERSITY OF SOUTHERN CALIFORNIA

CALIFORNIA INSTITUTE OF TECHNOLOGY

UNIVERSITY OF CALIFORNIA

STANFORD UNIVERSITY

UNIVERSITY OF TOKYO

MONTANA STATE UNIVERSITY

UNIVERSITY OF UTAH

UNIVERSITY OF NEVADA

WASHINGTON STATE UNIVERSITY

NEW MEXICO STATE UNIVERSITY

UNIVERSITY OF WASHINGTON

OREGON STATE UNIVERSITY

UNIVERSITY OF OREGON

OSAKA UNIVERSITY

AMERICAN MATHEMATICAL SOCIETY
NAVAL WEAPONS CENTER

Printed in Japan by International Academic Printing Co., Ltd., Tokyo, Japan 


\section{Pacific Journal of Mathematics}

\section{Vol. 63, No. $1 \quad$ March, 1976}

Ralph Artino, Gevrey classes and hypoelliptic boundary value problems ....... 1

B. Aupetit, Caractérisation spectrale des algèbres de Banach commutatives .... 23

Leon Bernstein, Fundamental units and cycles in the period of real quadratic

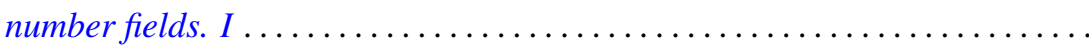

Leon Bernstein, Fundamental units and cycles in the period of real quadratic number fields. II.................................... 63

Robert F. Brown, Fixed points of automorphisms of compact Lie groups ........

Thomas Ashland Chapman, Concordances of noncompact Hilbert cube

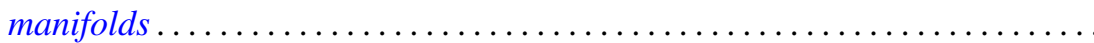

William C. Connett, V and Alan Schwartz, Weak type multipliers for Hankel

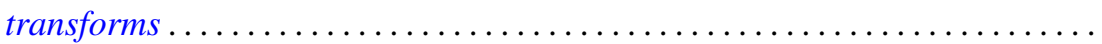

John Wayne Davenport, Multipliers on a Banach algebra with a bounded approximate identity .....................................

Gustave Adam Efroymson, Substitution in Nash functions ................ 137

John Sollion Hsia, Representations by spinor genera ..................

William George Kitto and Daniel Eliot Wulbert, Korovkin approximations in

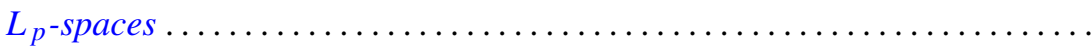

Eric P. Kronstadt, Interpolating sequences for functions satisfying a Lipschitz. condition ...........................................

Gary Douglas Jones and Samuel Murray Rankin, III, Oscillation properties of certain self-adjoint differential equations of the fourth order...

Takaŝi Kusano and Hiroshi Onose, Nonoscillation theorems for differential

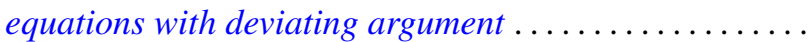

David C. Lantz, Preservation of local properties and chain conditions in commutative group rings. ...

Charles W. Neville, Banach spaces with a restricted Hahn-Banach extension property....

Norman Oler, Spaces of discrete subsets of a locally compact group ...

Robert Olin, Functional relationships between a subnormal operator and its minimal normal extension.

Thomas Thornton Read, Bounds and quantitative comparison theorems for nonoscillatory second order differential equations ...... .

Robert Horace Redfield, Archimedean and basic elements in completely distributive lattice-ordered groups...

Jeffery William Sanders, Weighted Sidon sets

Aaron R. Todd, Continuous linear images of pseudo-complete linear topological spaces.

J. Jerry Uhl, Jr., Norm attaining operators on $L^{1}[0,1]$ and the Radon-Nikodým property.

William Jennings Wickless, Abelian groups in which every endomorphism is a left multiplication. 